\title{
Identification of a Radical Formed in the Reaction Mixtures of Ram Seminal Vesicle Microsomes with Arachidonic Acid Using High Performance Liquid Chromatography-Electron Spin Resonance Spectrometry and High Performance Liquid Chromatography- Electron Spin Resonance-Mass Spectrometry
}

\author{
Katsuyuki Minakata ${ }^{1,2}$ and Hideo Iwahashi, ${ }^{1, *}$ \\ ${ }^{1}$ Department of Chemistry, Wakayama Medical University, 580 Mikazura, Wakayama 641-0011, Japan \\ ${ }^{2}$ Morinomiya College of Medical Arts and Sciences, 4-1-8 Nakamoto Higashinariku Osaka 537-0022, Japan
}

Received 29 September, 2009; Accepted 6 November, 2009

\begin{abstract}
Summary The reaction of ram seminal vesicle (RSV) microsomes with arachidonic acid (AA) was examined using electron spin resonance (ESR), high performance liquid chromatography-electron spin resonance spectrometry (HPLC-ESR), and high performance liquid chromatography-electron spin resonance-mass spectrometry (HPLC-ESR-MS) combined use of spin trapping technique. A prominent ESR spectrum $\left(\alpha^{\mathrm{N}}=1.58 \mathrm{mT}\right.$ and $\left.\alpha^{\mathrm{H}} \beta=0.26 \mathrm{mT}\right)$ was observed in the complete reaction mixture of ram seminal vesicle microsomes with arachidonic acid containing $2.0 \mathrm{mg}$ protein/ml ram seminal vesicle (RSV) microsomal suspension, $0.8 \mathrm{mM}$ arachidonic acid, $0.1 \mathrm{M}$ 4-POBN, and $24 \mathrm{mM}$ tris/HCl buffer (pH 7.4). The ESR spectrum was hardly observed for the complete reaction mixture without the RSV microsomes. The formation of the radical appears to be catalyzed by the microsomal components. In the absence of AA, the intensity of the ESR signal decreased to $16 \pm 15 \%$ of the complete reaction mixture, suggesting that the radical is derived from AA. For the complete reaction mixture with boiled microsomes, the intensity of the ESR signal decreased to $49 \pm 4 \%$ of the complete reaction mixture. The intensity of the ESR signal of the complete reaction mixture with indomethacin decreased to $74 \pm 20 \%$ of the complete reaction mixture, suggesting that cyclooxygenese partly participates in the reaction. A peak was detected on the elution profile of HPLC-ESR analysis of the complete reaction mixture. To determine the structure of the peak, an HPLC-ESR-MS analysis was performed. The HPLC-ESR-MS analysis of the peak showed two prominent ions, $\mathrm{m} / \mathrm{z} 266$ and $\mathrm{m} / \mathrm{z} 179$, suggesting that the peak is a 4-POBN/pentyl radical adduct. An HPLC-ESR analysis of the authentic 4-POBN/pentyl radical adduct comfirmed the identification.
\end{abstract}

Key Words: radical, arachidonic acid, peroxidation, COX, ESR

Introduction

Cyclooxygenase (COX) catalyzes the primary step in the

*To whom correspondence should be addressed.

Tel: +81-73-441-0772 Fax: +81-73-441-0772

E-mail: chem1@wakayama-med.ac.jp metabolism of unesterified arachidonic acid to bioactive prostanoids $[1,2]$. The prostanoids participate in physiological processes, such as synaptic transmission, neurotransmitter release and cerebral blood flow regulation $[3,4]$. The reaction of tissues to certain stresses such as inflammation or injuries is also caused by prostanoids. This metabolic pathway has been shown to play an important role in neurological and neurodegenerative diseases, such as stroke, 
epilepsy, and Alzheimer's disease [3,4].

The mechanism of prostaglandin synthesis predicts the formation of free radicals derived from the substrate fatty acid [5]. Indeed, Mason et al. observed a characteristic ESR spectrum for the reaction mixture of ram seminal vesicle microsomes with arachidonic acid in the presence of the spin trap, 2-methyl-2-nitrosopropane [6], suggesting that a carbon-centered free radical forms. Substitution of arachidonic acid by octadeuterated $(5,6,8,9,11,12,14,15)$ arachidonic acid confirmed that the radical is derived from arachidonic acid. Furthermore, the ESR spectrum showed that the arachidonic acid-derived radical is bound to the spin trap at one of the eight deuterated positions [7].

Free radicals have been successfully detected and identified using ESR spectroscopy combined with spin trapping technique. High performance liquid chromatographyelectron spin resonance spectrometry (HPLC-ESR) [8-11] and high performance liquid chromatography-electron spin resonance-mass spectrometry (HPLC-ESR-MS) [12] have also been employed for detection and identification of the radical adducts. To identify the radical formed in the reaction mixture of ram seminal vesicle microsomes with arachidonic acid, the HPLC-ESR and HPLC-ESR-MS were performed. In this paper, pentyl radical is detected and identified in the reaction mixtures of ram seminal vesicle microsomes with arachidonic acid using ESR, HPLC-ESR, and HPLC-ESR-MS combined use of spin trapping technique.

\section{Materials and Methods}

\section{Chemicals}

$\alpha$-(4-Pyridyl-1-oxide)- $N$-tert-butylnitrone (4-POBN), a spin-trapping reagent was purchased from Tokyo Kasei Kogyo, Ltd. (Tokyo, Japan). Arachidonic acid and indomethacin were from Wako Pure Chemical Industries, Ltd. (Osaka, Japan). Pentylhydrazine oxalate was synthesized according to the method of Gever and Hayes [13]. All other chemicals used were of analytical grade.

\section{Preparation of ram seminal vesicle microsomes}

The ram seminal vesicles (obtained from a local slaughterhouse) were freed of extraneous tissue, cut into small pieces, and homogenized in $0.25 \mathrm{M}$ sucrose (tissue to sucrose ratio of 1:9) using T25 digital ULTRA-TURRAX high-performance disperser (IKA-WERKE GMBH \& CO. KG, Staufen, Germany). The homogenate was centrifuged at $16,000 \mathrm{~g}$ for $30 \mathrm{~min}$ at $4^{\circ} \mathrm{C}$. The supernatant fraction was then centrifuged at $120,000 \mathrm{~g}$ for $30 \mathrm{~min}$ at $4^{\circ} \mathrm{C}$. The pellet was suspended in $0.25 \mathrm{M}$ sucrose. Protein concentration of the suspension was $4.8 \mathrm{mg} / \mathrm{ml}$. It was kept at $-80^{\circ} \mathrm{C}$ before use.
The complete reaction mixture of ram seminal vesicle microsomes with arachidonic acid

The complete reaction mixture contained $0.1 \mathrm{M} 4-\mathrm{POBN}$, $2.0 \mathrm{mg}$ protein $/ \mathrm{ml}$ ram seminal vesicle microsomes and $0.8 \mathrm{mM}$ arachidonic acid in $24 \mathrm{mM}$ tris buffer ( $\mathrm{pH}$ 7.4). The reaction was started by adding ram seminal vesicle and performed for the ESR, HPLC-ESR, and HPLC-ESR-MS experiments for $60 \mathrm{~min}$ at $37^{\circ} \mathrm{C}$.

\section{Preparation of 4-POBN/pentyl radical adduct}

The reaction mixture contained $0.1 \mathrm{M}$ 4-POBN, $4 \mathrm{mg} / \mathrm{ml}$ pentylhydrazine oxalate, $0.2 \mathrm{mM} \mathrm{CuCl}_{2}$ and $45 \mathrm{mM}$ carbonate buffer ( $\mathrm{pH} 10.0)$ in a total volume of $2 \mathrm{ml}$. The reaction was started by adding $\mathrm{CuCl}_{2}$ after bubbling nitrogen gas. It was performed for $120 \mathrm{~min}$ under an air atmosphere. In order to purify 4-POBN/pentyl radical adduct, the reaction mixture was applied to the HPLC-ESR.

\section{ESR measurements}

The ESR spectra were obtained using a model JES-FR30 Free Radical Monitor (JEOL Ltd., Tokyo, Japan). Aqueous samples were aspirated into a Tefron tube centered in a microwave cavity. Operating conditions of the ESR spectrometer were: power, $4 \mathrm{~mW}$; modulation width, $0.1 \mathrm{mT}$; sweep time, $4 \mathrm{~min}$; sweep width, $10 \mathrm{mT}$; time constant, $0.3 \mathrm{~s}$. Magnetic fields were calibrated by the splitting of $\mathrm{MnO}\left(\Delta \mathrm{H}_{3-4}=8.69 \mathrm{mT}\right)$.

\section{HPLC-ESR chromatography}

An HPLC used in the HPLC-ESR consisted of a model 7125 injector (Reodyne, Cotari, CA) and a model 655A-11 pump with a model L-5000 LC controller (Hitachi Ltd., Ibaragi, Japan). A semi-preparative column (300 mm long $\times$ $10 \mathrm{~mm}$ i.d.) packed with TSKgel ODS-120T (TOSOH Co., Tokyo, Japan) was used. Flow rate was $2.0 \mathrm{ml} / \mathrm{min}$ throughout the experiments. For the HPLC-ESR, two solvents were used: solvent A, $50 \mathrm{mM}$ acetic acid; solvent B, $50 \mathrm{mM}$ acetic acid/acetonitrile (20:80, v/v). A following combination of isocratic and linear gradient was used: $0-40 \mathrm{~min}$, $100-20 \%$ A (linear gradient); 40-60 min, 80\% B (isocratic). The eluent was introduced into a model JES-FR30 Free Radical Monitor. The ESR spectrometer was connected to the HPLC with a Teflon tube, which passed through the center of the ESR cavity. The operating conditions of the ESR spectrometer were: power, $4 \mathrm{~mW}$; modulation width, $0.2 \mathrm{mT}$; time constant, $1 \mathrm{~s}$. The magnetic field was fixed at the third peak in the doublet-triplet ESR spectrum $\left(\alpha^{\mathrm{N}}=\right.$ $1.58 \mathrm{mT}$ and $\alpha^{\mathrm{H}} \beta=0.26 \mathrm{mT}$ ) of the 4-POBN radical adduct.

\section{HPLC-ESR-MS chromatography}

HPLC and ESR conditions were as described in the HPLC-ESR. The operating conditions of the mass spectrometer were: nebulizer, $180^{\circ} \mathrm{C}$; aperture $1,120^{\circ} \mathrm{C} ; \mathrm{N}_{2}$ 
controller pressure, $2.0 \mathrm{~kg} / \mathrm{cm}^{2}$ drift voltage, $70 \mathrm{~V}$; multiplier voltage, $1800 \mathrm{~V}$; needle voltage, $3000 \mathrm{~V}$; polarity, positive; resolution, 48 .

The mass spectra were obtained by introducing the eluent from the ESR detector into the LC-MS system just before the peaks were eluted. The flow rate was kept at $50 \mu \mathrm{l} / \mathrm{min}$ while the eluent was introducing into the mass spectrometer.

\section{Results}

ESR measurements of the reaction mixtures of ram seminal vesicle microsomes with arachidonic acid

An ESR spectrum of the complete reaction mixture of ram seminal vesicle microsomes with arachidonic acid was measured (Fig. 1). A prominent ESR spectrum $\left(\alpha^{\mathrm{N}}=\right.$ $1.58 \mathrm{mT}$ and $\alpha^{\mathrm{H}} \beta=0.26 \mathrm{mT}$ ) was observed in the complete reaction mixture (Fig. 1A). The ESR spectrum was hardly observed for the complete reaction mixture without ram seminal vesicle microsomes (Fig. 1B, Table 1). In the absence of arachidonic acid, the intensity of the ESR signal decreased to $16 \pm 15 \%(n=3)$ of the complete reaction mixture (Fig. 1C, Table 1). For the complete reaction mixture with boiled microsomes, the ESR signal decreased to $49 \pm 4 \% \quad(n=3)$ of the complete reaction mixture (Fig. 1D, Table 1). For the complete reaction mixture with indomethacin, the ESR signal decreased to $74 \pm 20 \%(n=3)$ of the complete reaction mixture (Fig. 1E, Table 1).

HPLC-ESR and HPLC-ESR-MS analyses of the reaction mixture of ram seminal vesicle microsomes with arachidonic acid

The HPLC-ESR analyses were performed for the complete reaction mixture of ram seminal vesicle microsomes with arachidonic acid (Fig. 2). A prominent peak was separated on the HPLC-ESR elution profile (Fig. 2A). The retention times of the peak was $49.4 \mathrm{~min}$.

To determine the structure of the peak compound, an HPLC-ESR-MS analysis was performed. The HPLC-ESRMS analysis of the peak compound gave ions at $m / z 266$ and $m / z 179$ (Fig. 3). The ion $m / z 266$ corresponds to the protonated molecule of 4-POBN/pentyl radical adduct, $[\mathrm{M}+\mathrm{H}]^{+\bullet}$. A fragment ion at $\mathrm{m} / \mathrm{z} 179$ corresponds to the loss of $\left(\mathrm{CH}_{3}\right)_{3} \mathrm{C}(\mathrm{O}) \mathrm{N}$ from the protonated molecules.

In order to confirm the chemical structure of the peak compound, HPLC-ESR analyses were performed for the reaction mixture of pentylhydrazine with $\mathrm{Cu}^{2+}$. It is well known that pentyl radical forms in the reaction mixture of pentylhydrazine with $\mathrm{Cu}^{2+}(11)$. An HPLC-ESR analysis of the reaction mixture of pentylhydrazine with $\mathrm{Cu}^{2+}$ gave a peak with almost the same retention time as the peak compound (Fig. 2B). When the peak fraction in the reaction mixture of pentylhydrazine with $\mathrm{Cu}^{2+}$ was mixed with the complete reaction mixture of ram seminal vesicle micro-

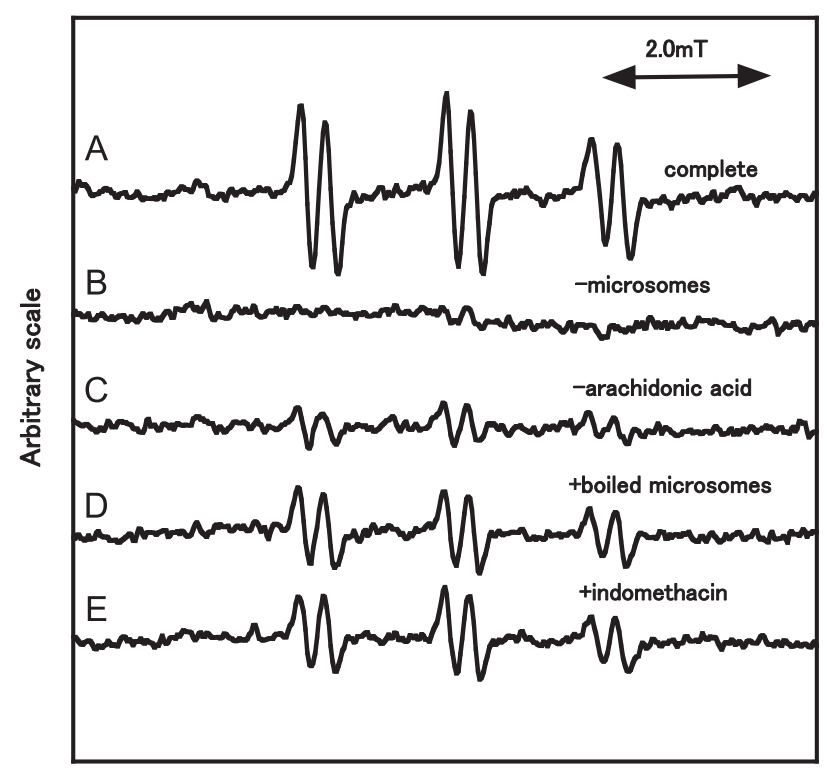

Fig. 1. ESR spectra of the reaction mixtures of ram seminal vesicle microsomes with arachidonic acid. The reaction and ESR conditions were as described in Materials and Methods. Total volume of the reaction mixtures was $200 \mu \mathrm{l}$. A, a complete reaction mixture of sam seminal vesicle microsomes with arachidonic acid; B, same as in A except that microsomes were omitted; $\mathrm{C}$, same as in $\mathrm{A}$ except that arachidonic acid was omitted; D, a complete reaction mixture with boiled microsomes; $\mathrm{E}$, same as in A except that indomethacin $(2 \mathrm{mM})$ was added.

Table 1. ESR peak heights of the reactions of ram seminal vesicles with arachidonic acid under several conditions

\begin{tabular}{lc}
\hline Conditions & $\%$ control \\
\hline -Microsomes & $12 \pm 3$ \\
-Arachidonic acid & $16 \pm 15$ \\
+Boiled microsomes & $49 \pm 4$ \\
+Indomethacin $(2 \mathrm{mM})$ & $74 \pm 20$ \\
\hline
\end{tabular}

Results are means \pm SD of three experiments. ESR measurement and reaction were performed as described in Materials and Methods. The control is the complete reaction mixture.

somes with arachidonic acid, the peak height of the peak compound increased (Fig. 2C), suggesting that the peak compound and 4-POBN/pentyl radical adduct are identical.

\section{Discussion}

In this study, the reaction of ram seminal vesicle microsomes with arachidonic acid was examined using ESR, HPLC-ESR and HPLC-ESR-MS combined use of spin trapping technique. A prominent ESR spectrum $\left(\alpha^{\mathrm{N}}=1.58\right.$ $\mathrm{mT}$ and $\alpha^{\mathrm{H}} \beta=0.26 \mathrm{mT}$ ) was observed in the complete reaction mixture. The ESR spectrum was hardly observed 


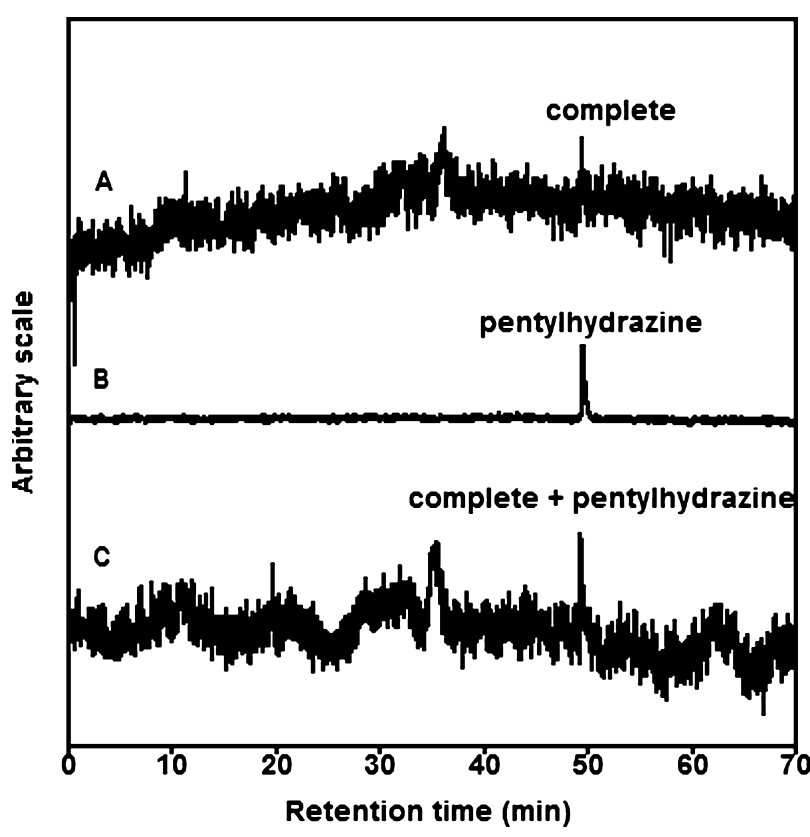

Fig. 2. HPLC-ESR analyses of the reaction mixtures of ram seminal vesicle microsomes with arachidonic acid. The reaction and HPLC-ESR conditions were as described in Materials and Methods. Total volume of the complete reaction mixtures was $10 \mathrm{ml}$. A, $10 \mathrm{ml}$ of complete reaction mixture; $\mathrm{B}, 10 \mu \mathrm{l}$ of $4-\mathrm{POBN} /$ pentyl radical adducts; $\mathrm{C}, 10 \mathrm{ml}$ of complete reaction mixture mixed with $10 \mu \mathrm{l}$ of 4-POBN/pentyl radical adducts diluted by one fifteenth.

for the complete reaction mixture without ram seminal vesicle microsomes. The ram seminal vesicle microsomes could be essential for the radical formation. The ESR spectrum was hardly observed in the absence of arachidonic acid, suggesting that the radical is derived from arachidonic acid.

HPLC-ESR and HPLC-ESR-MS analyses showed that 4-POBN/pentyl radical adduct forms in a reaction mixture of ram seminal vesicle microsomes with arachidonic acid. A possible reaction path for the formation of the pentyl radical is shown (Scheme 1). The prostaglandin endoperoxide $\mathrm{H}$ synthases (PGHSs) exhibit two different but complementary enzymatic activities [1,5]: (i) a COX (bis-oxygenase) which catalyzes the formation of prostaglandin $\mathrm{G}_{2}\left(\mathrm{PGG}_{2}\right)$ from arachidonic acid and two molecules of $\mathrm{O}_{2}$ and (ii) a peroxidase which facilitates the two-electron reduction of the 15-hydroperoxyl group of $\mathrm{PGG}_{2}$. Rota et al. reported the cytochrome-P450-catalyzed one electron reductive homolytic decomposition of $\mathrm{LOOH}$ [14]. The one electron reductive homolytic decomposition of $\mathrm{LOOH}$ is also catalyzed by the other compounds such as hemoglobin, myoglobin, and cytochrome c [15-17]. This reaction yields radical intermediates alkoxyl radical (LO').

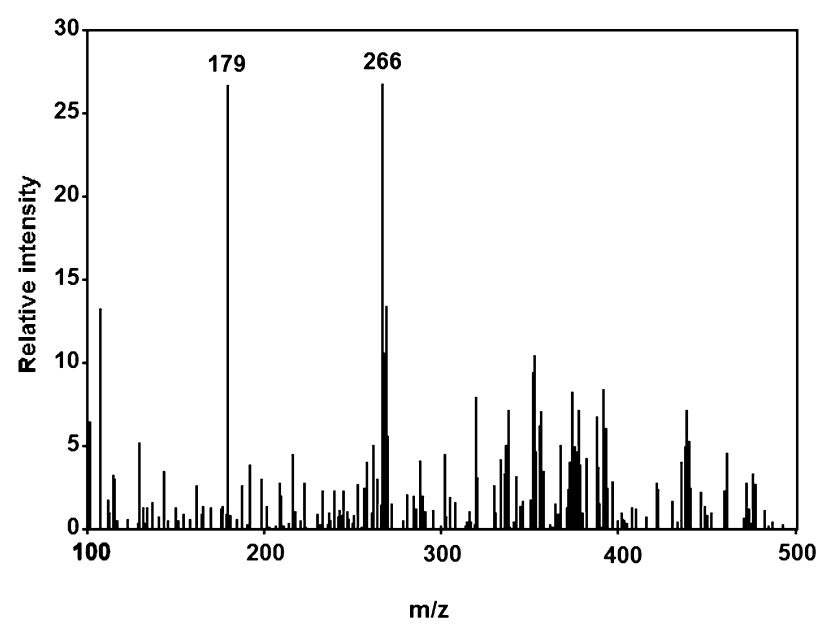

Fig. 3. HPLC-ESR-MS analysis. The reaction and HPLC-ESRMS conditions were as described in Materials and Methods. Total volume of the reaction mixtures was $10 \mathrm{ml}$.

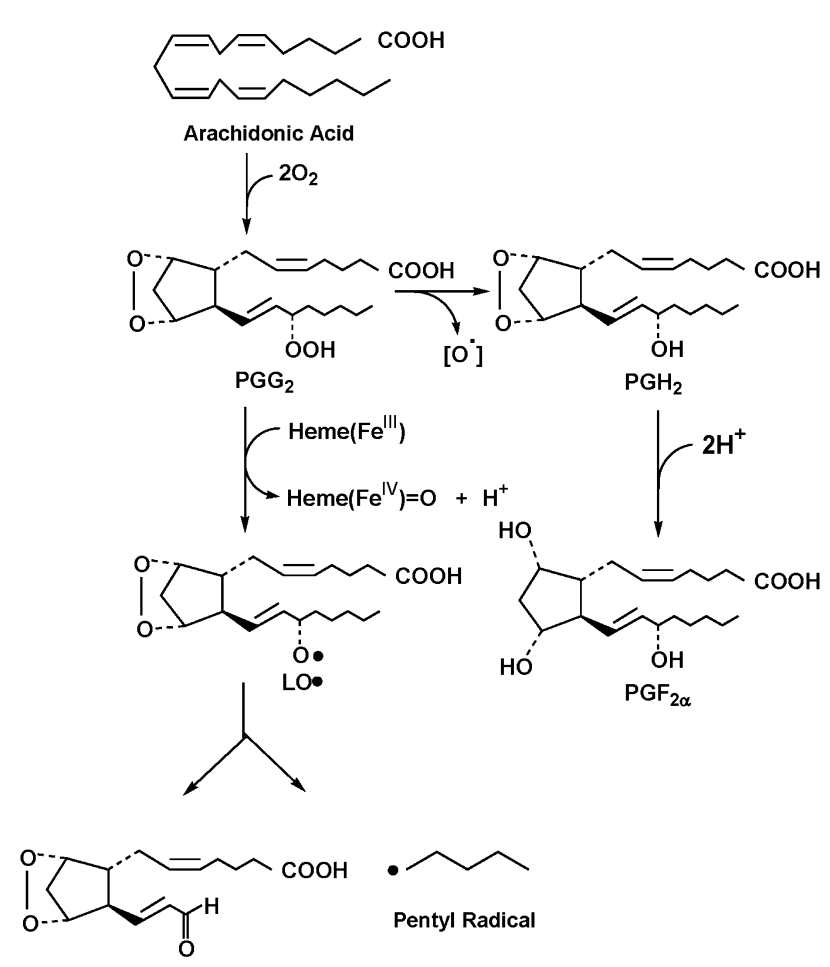

Scheme 1. A possible reaction path for the formation of pentyl radical.

$$
\operatorname{Heme}\left(\mathrm{Fe}^{\mathrm{III}}\right)+\mathrm{PGG}_{2} \rightarrow \operatorname{Heme}\left(\mathrm{Fe}^{\mathrm{IV}}\right)=\mathrm{O}+\mathrm{H}^{+}+\mathrm{LO}^{*}
$$

$\beta$-Scission of the LO possibly forms the pentyl radical (Scheme 1). Mason et al. observed a characteristic ESR spectrum for the reaction mixture of ram seminal vesicle microsomes with arachidonic acid in the presence of the spin trap, 2-methyl-2-nitrosopropane [6], suggesting that a 
carbon-centered free radical forms. Substitution of arachidonic acid by octadeuterated $(5,6,8,9,11,12,14,15)$ arachidonic acid confirmed that the radical is derived from arachidonic acid. Furthermore, the ESR spectrum showed that the arachidonic acid derived radical is bound to the spin trap at one of the eight octadeuterated positions [7]. The pentyl radicals detected in this paper is obviously different from the one detected by Mason et al. Different spin trap reagents seem to trap different radicals even in a similar reaction.

Recently, 8-iso-prostaglandin $\mathrm{F}_{2 \alpha}\left(\mathrm{PGF}_{2 \alpha}\right)$, a major isoprostane generated through the non-enzymatic peroxidation of arachidonic acid, has been shown to be a reliable indicator of oxidative stresses in various clinical conditions [18]. Reports have shown that F2-isoprostanes are authentic biomarkers of lipid peroxidation and can be used as potential in vivo indicators of oxidant stress, as well as in evaluations of antioxidants or drugs for their free radical-scavenging properties [8]. The $\mathrm{PGF}_{2 \alpha}$ is formed from $\mathrm{PGG}_{2}$ via prostaglandin endoperoxide $\mathrm{H}_{2}\left(\mathrm{PGH}_{2}\right)$. The pentyl radical detected in this paper is also form from $\mathrm{PGG}_{2}$. Thus, both pentyl radical and $\mathrm{PGF}_{2} \alpha$ are complementary biomarkers of lipid peroxidation.

\section{References}

[1] Smith, W.L. and Murphy, R.C.: The eicosanoids: cyclooxygenase, lipoxygenase, and epoxygenase pathways, in Biochemistry of Lipids, Lipoproteins and Membranes, 5th edition, eds. By Vance, D.E. and Vance, J.E., Elsevier, Amsterdam, pp. 331-362, 2008.

[2] Smith, W.L., DeWitt, D.L., and Garavito, R.M.: Cyclooxygenase: structural, cellular, and molecular biology. Annu. Rev. Biochem., 69, 145-182, 2000.

[3] Kam, P.C. and See, A.U.: Cyclo-oxygenase isoenzymes: physiological and pharmacological role. Anaesthesia, 55, 442-449, 2000.

[4] Katori, M. and Majima, M.: Cyclooxygenase-2: its rich diversity of roles and possible application of its selective inhibitors. Inflamm. Res., 49, 367-392, 2000.

[5] Rouzer, C.A. and Marnett, L.J.: Mechanism of free radical oxygenation of polyunsaturated fatty acids by cyclooxygenases. Chem. Rev., 103, 2239-2304, 2003.

[6] Mason, R.P., Kalyanaraman, B., Tainer, B.E., and Eling, T.E.: A carbon-centered free radical intermediate in the prostaglandin synthetase oxidation of arachidonic acid. $J$.
Biol. Chem., 255, 5019-5022, 1980.

[7] Schreiber, J., Eling, T.E., and Mason, R.P.: The oxidation of arachidonic acid by the cyclooxygenase activity of purified prostaglandin $\mathrm{H}$ synthase: spin trapping of a carbon-centered free radical intermediate. Arch. Biochem. Biophys., 249, 126136, 1986.

[8] Rokushika, S., Taniguchi, H., and Hatano, H.: Flow ESR detector for liquid chromatography of radicals. Anal. Lett., 8, 205-213, 1975.

[9] Makino, K. and Hatano, H.: Separation and characterization of short-lived radicals in DL-methionine aqueous solution by high speed liquid chromatograph equipped with ESR spectrometer. Chem. Lett., 119-122, 1979.

[10] Iwahashi, H., Ikeda, A., Negoro, Y., and Kido, R.: Detection of radical species in haematin-catalysed retinoic acid 5,6epoxidation by using h.p.l.c.-e.p.r. spectrometry. Biochem. J., 236, 509-514, 1986.

[11] Iwahashi, H., Albro, P.W., McGown, S.R., Tomer, K.B., and Mason, R.P.: Isolation and identification of $\alpha$-(4-pyridyl-1oxide)-N-tert-butylnitrone radical adducts formed by the decomposition of the hydroperoxides of linoleic acid, linolenic acid, and arachidonic acid by soybean lipoxygenase. Arch. Biochem. Biophys., 285, 172-180, 1991.

[12] Iwahashi, H., Parker, C.E., Mason, R.P., and Tomer, K.B.: Combined liquid chromatography/electron paramagnetic resonance spectrometry/electrospray ionization mass spectrometry for radical identification. Anal. Chem., 64, 2244-2252, 1992.

[13] Gever, G. and Hayes, K.: Alkylhydrazines. J. Org. Chem., 14, 813-818, 1949.

[14] Rota, C., Barr, D.P., Martin, M.V., Guengerich, F.P., Tomasi, A., and Mason, R.P.: Detection of free radicals produced from the reaction of cytochrome P-450 with linoleic acid hydroperoxide. Biochem. J., 328, 565-571, 1997.

[15] Iwahashi, H.: Some polyphenols inhibit the formation of pentyl radical and octanoic acid radical in the reaction mixture of linoleic acid hydroperoxide with ferrous ions. Biochem. J., 346, 265-273, 2000.

[16] Iwahashi, H., Nishizaki, K., and Takagi, I.: Cytochrome c catalyses the formation of pentyl radical and octanoic acid radical from linoleic acid hydroperoxide. Biochem. J., 361, 57-66, 2002.

[17] Iwahashi, H., Kumamoto, K., and Hirai, T.: The formation of the 7-carboxyheptyl radical from 13-hydroperoxy-9,11octadecadienoic acid catalyzed by hemoglobin and myoglobin under anaerobic conditions. J. Biochem., 133, 679-685, 2003. 